extent, duration, concurrent immunomodulatory therapy) predicted steroid free remission at 12 months.

Conclusions A raised basal calprotectin was associated with a lower probability of steroid free clinical remission with both anti-TNF and anti-integrin biological therapy in UC. None of the other clinical variables including BMI at baseline predicted steroid free clinical remission at 1 year.

\section{PWE-061 PREDICTORS OF REMISSION TO VEDOLIZUMAB THERAPY IN A COMBINED INFLAMMATORY BOWEL DISEASES COHORT}

${ }^{1}$ Sreedhar Subramanian, ${ }^{2}$ Paula Mcpartland, ${ }^{2}$ Susie Dodd, 'Daniel Storey, 'Alan Steel, ${ }^{1}$ Martyn Dibb, 'Paul Collins, ${ }^{2}$ Chris Probert, ${ }^{1} E d$ Derbyshire, ${ }^{2}$ Rebecca Davis*. ${ }^{1}$ Royal Liverpool University Hospital, Liverpool, UK; ${ }^{2}$ University of Liverpool, Liverpool, UK

\subsection{6/gutjnl-2018-BSGAbstracts. 193}

Introduction The response to the anti-integrin- $\alpha_{4} \beta_{7}$ antibody, vedolizumab, is variable in both Crohn's disease and ulcerative colitis. Apart from prior exposure to anti-TNF agents, there are very few clinical predictors of response to vedolizumab therapy. Previous studies have shown that clinical and biological variables such as body mass index (BMI), baseline disease activity and concurrent immunosuppression influence response to anti-TNF agents but such data are not available for vedolizumab. We sought to evaluate the role of clinical and biological variables as predictors of response to vedolizumab.

Methods We conducted a retrospective single centre retrospective cohort study of all patients commencing vedolizumab therapy. Clinical response was defined as a reduction of 3 points from baseline for Harvey-Bradshaw index (HBI) and 2 points for simple clinical colitis activity index (SCCAI). Clinical remission was defined as $\mathrm{HBI}<5$ and SCCAI $<2$. Response and remission was evaluated at 6 and 12 months. A multi-variate logistic regression was performed to analyse the effect of BMI, baseline calprotectin, prior anti-TNF exposure, disease type (UC or $\mathrm{CD}$ ) and concomitant IS on response and remission to vedolizumab.

Results A total of 95 patients commenced vedolizumab therapy during the study period (2014-17). Of these, 11 patients $(11.5 \%)$ had recently commenced therapy and a further 20 patients (21\%) were excluded (Surgery, $n=4$, adverse reaction, $\mathrm{n}=4$, primary non-response, $\mathrm{n}=5$ and insufficient data, $\mathrm{n}=7$ ). Of these, 64 patients had disease activity data at 6 months and 45 patients at 12 months. The pooled steroid free remission rates at 6 and 12 months were $45 \%$ and $32 \%$ respectively. Baseline clinical factors including BMI, smoking status, concurrent immunomodulatory or steroid therapy were not associated with steroid free remission at 6 and 12 months. Prior anti-TNF failure did not influence clinical remission at 6 months (OR 0.7, 95\% CI 0.26-1.88, p=0.4) or 12 months (OR 1.24, 95\% CI 0.44-3.46, p=0.6). Patients with UC were more likely to be in steroid free remission at 6 (OR 2.45, 95\% CI 0.89-6.74, $\mathrm{p}=0.08$ ) and 12 months (OR 3.5, 95\% CI $1.2-10.6, p=0.02)$. Neither baseline calprotectin nor a reduction in calprotectin at 2 months predicted steroid free remission at 6 or 12 months.

Conclusions In a mixed IBD cohort treated with vedolizumab, patients with UC were more likely to be in steroid free remission at 6 and 12 months. None of the other clinical and biological variables were associated with steroid free remission.
PWE-062 FIRST CLINICAL EXPERIENCE OF PANENTERIC CAPSULE ENDOSCOPY USING THE PILLCAM CROHN'S CAPSULE

${ }^{1}$ David Tai ${ }^{*},{ }^{1}$ Victoria Thurston, ${ }^{1}$ Ailish Healy, ${ }^{2}$ Sara Koo, ${ }^{2}$ Clare Parker, ${ }^{2}$ Simon Panter, ${ }^{1}$ Melissa Hale, ${ }^{1}$ Reena Sidhu, ${ }^{1}$ Mark McAlindon. ${ }^{1}$ Sheffield Teaching Hospitals NHS Trust, Sheffield, UK; ${ }^{2}$ South Tyneside NHS Foundation Trust, Tyne and Wear, UK

\subsection{6/gutjnl-2018-BSGAbstracts. 194}

Introduction Guidelines support endoscopic assessment of mucosal healing in Crohn's disease before a change in therapy. [Gomollon, J Crohn's Colitis 2016] A recent study has shown that the PillCam Crohn's (PCC, Medtronic, Dublin, Ireland) has a better diagnostic yield than ileocolonoscopy [Leighton, Gastrointest Endosc 2017] and that colon capsule (for which the same bowel preparation is used) is better tolerated [Ojidu, European J Gastroenterol Hepatol, in press]. We report the first experience of PCC in routine clinical practice.

Methods Data was collected prospectively in Sheffield and South Tyneside hospitals. Montreal classification was used (ileal:L1; colonic: L2; ileocolonic: L3; upper GI: L4; B1: nonstricturing/penetrating; B2: stricturing; B3: penetrating). All patients passed an Agile patency capsule (Medtronic).

Results Eighteen patients (median age 35 years, 38.9\% male, known Crohn's in 83\%) had PCC. Indications were: symptom assessment $(77.8 \%)$, assess response to treatment $(11.1 \%)$, consideration of stepping down therapy (16.7\%). Patients with established Crohn's had L1 (53.3\%), L2 (13.3\%), and L3 disease $(33.3 \%)$ which was uncomplicated (40\%), stricturing $(46.7 \%)$ and penetrating (13.3\%). Patients were on medical treatment in $73.3 \%$. PCC changed staging of disease in $33 \%$ of cases ( $\mathrm{L} 1$ to $\mathrm{L} 3 \mathrm{n}=1, \mathrm{~B} 2$ to $\mathrm{B} 1 \mathrm{n}=3$ and $\mathrm{B} 1$ to $\mathrm{B} 2 \mathrm{n}=1$ ). One of three patients with suspected Crohn's disease subsequently had endoscopic confirmation (L3 B1). PCC was normal (5/18), revealed L1 disease alone (8/18), L2 disease alone $(1 / 18)$ and L3 disease (5/18). There were three incomplete procedures, all with an otherwise normal visualised colon. No capsule retentions occurred. Follow up data was available in 11 patients. Of eight patients with symptoms, five had active disease and three no or minimal activity. Of the five, three had a step-up in treatment, one had adalimumab temporarily suspended due to a perianal abscess and management continues to be discussed in one patient on maximal medical therapy. Other causes of symptoms were sought in the patients with inactive disease. Three patients had no symptoms, one had active disease and a step up in treatment, two had no or minimal activity of whom one continued therapy due to poor prognostic factors and one was already on no treatment (PCC being performed to provide supportive evidence of a diagnosis made in childhood). No patient known to have Crohn's has been referred for further small bowel imaging or colonoscopy. Conclusion PCC provides a single visit assessment of both small and large bowel which was useful in guiding patient management without complications.

\section{PWE-063 ALTERATIONS IN CORTICAL THICKNESS AND GREY MATTER VOLUME IN PATIENTS WITH ACTIVE CROHN'S DISEASE}

${ }^{1}$ Gordon Moran*, ${ }^{1,2}$ Gita Thapaliya, ${ }^{2}$ Sally Eldeghaidy, ${ }^{1}$ Shellie Jean Radford, ${ }^{1}$ Melanie Lingaya, ${ }^{1}$ Yirga Falcone, ${ }^{2}$ Sue Francis. ${ }^{1}$ Nottingham Digestive Disease Centre, University of Nottingham, Nottingham, UK; ${ }^{2}$ Sir Peter Mansfield Imaging Centre, University of Nottingham, Nottingham, UK

10.1136/gutjnl-2018-BSGAbstracts. 195 